\title{
DEVELOPMENT OF MULTI-WAVELENGTH RAMAN LIDAR AND ITS APPLICATION ON AEROSOL AND CLOUD RESEARCH
}

\author{
Dong Liu ${ }^{1}$, Yingjian Wang ${ }^{1,2}$, Zhenzhu Wang ${ }^{1}$, Zongming Tao ${ }^{3}$, Decheng $\mathrm{Wu}^{1,4}$, Bangxin Wang ${ }^{1}$, \\ Zhiqing Zhong ${ }^{1}$ and Chenbo Xie ${ }^{1}$ \\ ${ }^{1}$ Key Laboratory of Atmospheric Composition and Optical Radiation, Anhui Institute of Optics and Fine \\ Mechanics, Chinese Academy of Sciences, Hefei, Anhui 230031, China \\ ${ }^{2}$ University of Science and Technology of China, Hefei, Anhui 230031, China \\ ${ }^{3}$ Department of Basic Sciences, New Star Institute of Applied Technology, Hefei, Anhui 230031,China \\ ${ }^{4}$ Department of Atmospheric Sciences, University of Wyoming, WY 82070, USA \\ *Email:dliu@aiofm.ac.cn
}

\begin{abstract}
A movable multi-wavelength Raman lidar (TMPRL) was built in Hefei, China. Emitting with three wavelengths at 1064,532 , and $355 \mathrm{~nm}$, receiving three above Mie scattering signals and two nitrogen Raman signals at 386 and $607 \mathrm{~nm}$, and depolarization signal at $532 \mathrm{~nm}$, TMPRL has the capacity to investigate the height resolved optical and microphysical properties of aerosol and cloud. The retrieval algorithms of optical parameters base on Mie-Raman technique and the microphysical parameters based on Bayesian optimization method were also developed and applied to observed lidar data. Designing to make unattended operation and 24/7 continuous working, TMPRL has joined several field campaigns to study on the aerosol, cloud and their interaction researches. Some observed results of aerosol and cloud optical properties and the first attempt to validate the vertical aerosol size distribution retrieved by TMPRL and in-situ measurement by airplane are presented and discussed.
\end{abstract}

\section{INTRODUCTION}

Aerosol and cloud play an important role in modulating the balance of the radiation budget between the earth and its atmosphere directly and indirectly. They still show a big uncertainty on radiative forcing and climate studies[1]. Though variable means have been carried out to make observation of their optical and other properties, the vertical structures are still lack especial for their microphysics as well as the optical properties. Taking advantage of the profiling tool, a moveable multi-wavelength Raman lidar (TMPRL) was built to investigate the height resolved optical and microphysical properties of aerosol and cloud.
In this paper, the overall structures and the main specifications the lidar system are introduced. The retrieval algorithms of optical and microphysical parameters of aerosol and cloud are described. The observed results and the airplane validation experiment is presented and discussed. More results will be shown during the conference.

\section{METHODOLOGY}

\subsection{The TMPRL lidar system}

TMPRL is a powerful and continuous working lidar installed in a standard container with a window on the roof for easy transportation. A three-wavelength Nd:YAG laser was equipped as the transmitter. Three Mie scattering, two Raman scattering and one depolarization signals were collected by the telescope simultaneously, totaled in six receiving channels. A glass with a selfdesigned heater was covered on the roof window to get rid of the dew especially before the sunrise to ensure it could work under all weather conditions. The optical and mechanical structures of the lidar system were elaborated designed and installed to keep stable to meet the transportation request.

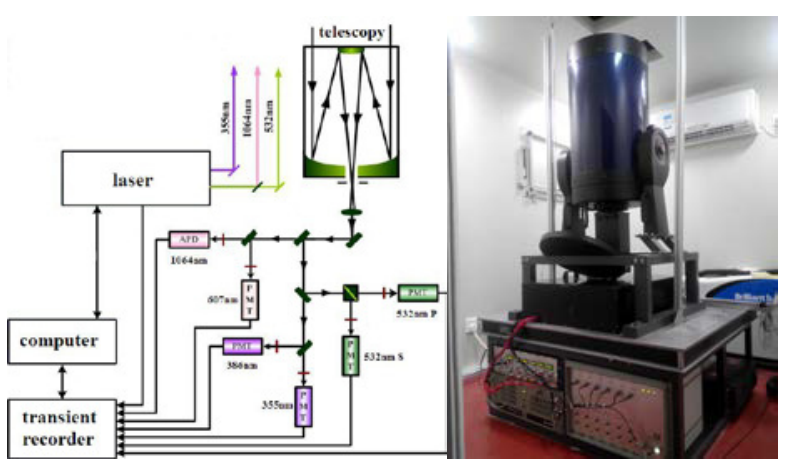

Fig.1 The diagram and photo of TMPRL 
Fig. 1 gives the system diagram and the photo of the TMPRL. Table 1 lists the main specifications of this lidar system. The Range-corrected signals of the six channels are shown in fig.2.

Table 1 Main specifications of TMPRL

\begin{tabular}{|l|l|}
\hline Laser & Nd:YAG (Quantel Brilliant B) \\
\hline Wavelength & $1064 \mathrm{~nm}, 532 \mathrm{~nm}, 355 \mathrm{~nm}$ \\
\hline Pulse energy & $280 \mathrm{~mJ}, 260 \mathrm{~mJ}, 160 \mathrm{~mJ}$ \\
\hline Repetition rate & $10 \mathrm{~Hz}$ \\
\hline Divergence & $0.5 \mathrm{mrad}$ \\
\hline Telescope & Cassegrain LX400-ACF-14" \\
\hline Detectors & APD for 1064nm, PMT for others \\
\hline Acquisition & Licel TR-20-160 \\
\hline
\end{tabular}

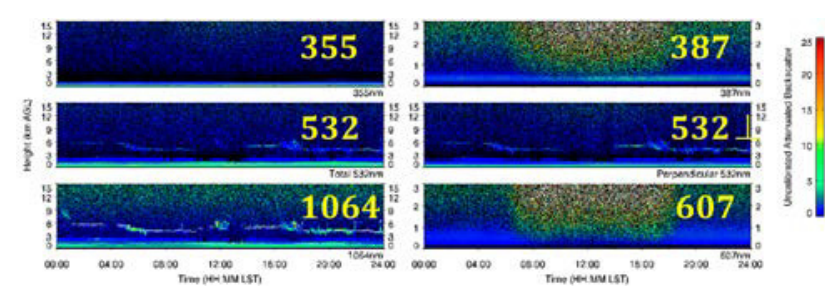

Fig.2 Range-corrected signals for 6 receiving channels

From fig.2, one can see TMPRL worked continuously for the whole day. The Mie signals including the perpendicular signal at $532 \mathrm{~nm}$ wavelength didn't show any day/night difference which indicated the signal-to-noise ratio of the Mie scattering are pretty good. For the Raman scattering signal is not the case due to their weaker cross section of the nitrogen stimulated by the 355 and $532 \mathrm{~nm}$ wavelength laser pulse.

\subsection{Optical parameters retrieval}

The common two component Mie scattering lidar equation can be expressed as:

$$
\begin{aligned}
P(r)= & P_{t} k r^{-2}\left[\beta_{m o l}(r)+\beta_{\text {aer }}(r)\right] \\
& \times \exp \left\{-2 \int_{0}^{r}\left[\alpha_{m o l}\left(r^{\prime}\right)+\alpha_{a e r}\left(r^{\prime}\right)\right] d r^{\prime}\right\}
\end{aligned}
$$

$P_{t}$ stands for emitted laser power, $P$ stands for received backscatter power in distance $r, \alpha$ and $\beta$ stands for extinction and backscattering coefficient, the subscript mol and aer stand for molecule and aerosol, $k$ is the system constant.
The backward iteration solution developed by Fernald(1984)[2] is selected for retrieving the extinction coefficient as shown in equation (2).

$$
\begin{aligned}
\alpha_{a}(r)=-\frac{S_{a}}{S_{m}} \cdot \alpha_{m}(r)+ & P(r) r^{2} \cdot \exp \left[2\left(\frac{S_{a}}{S_{m}}-1\right) \int_{r}^{r_{r}} \alpha_{m}\left(r^{\prime}\right) d r^{\prime}\right] \\
& \frac{P\left(r_{c}\right) r^{2}}{\alpha_{a}\left(r_{c}\right)+\frac{S_{a}}{S_{m}} \alpha_{m}\left(r_{c}\right)}+2 \int_{r}^{r_{c}} P\left(r^{\prime}\right) r^{\prime 2} \exp \left[2\left(\frac{S_{a}}{S_{m}}-1\right) \int_{r}^{r_{c}} \alpha_{m}\left(r^{\prime \prime}\right) d r^{\prime \prime}\right] d r^{\prime}
\end{aligned}
$$

For the Mie-Raman combined method, equation (3) and (4) gives the solution of extinction and backscattering coefficient[3].

$$
\begin{aligned}
\alpha_{\lambda_{0}}^{\text {aer }}(z)= & \frac{\frac{d}{d z} \ln \frac{N_{R}(z)}{P_{\lambda_{R}} z^{2}}-a_{\lambda_{0}}^{\text {mol }}(z)-a_{\lambda_{R}}^{\text {mol }}(z)}{1+\left(\frac{\lambda_{0}}{\lambda_{R}}\right)^{k(z)}} \\
\beta_{\lambda_{0}}^{\text {aer }}(z)= & {\left[\beta_{\lambda_{0}}^{\text {aer }}\left(z_{0}\right)+\beta_{\lambda_{0}}^{\text {mol }}\left(z_{0}\right)\right] \frac{p_{\lambda_{R}}\left(z_{0}\right) p_{\lambda_{0}}(z) N_{R}(z)}{p_{\lambda_{0}}\left(z_{0}\right) p_{\lambda_{R}}(z) N_{R}\left(z_{0}\right)} } \\
& \times \frac{\exp \left(-\int_{r_{0}}^{z}\left[\alpha_{\lambda_{R}}^{\text {aer }}\left(z^{\prime}\right)+\alpha_{\lambda_{R}}^{\text {mol }}\left(z^{\prime}\right)\right] d z^{\prime}\right)}{\exp \left(-\int_{r_{0}}^{z}\left[\alpha_{\lambda_{0}}^{\text {aer }}\left(z^{\prime}\right)+\alpha_{\lambda_{0}}^{\text {mol }}\left(z^{\prime}\right)\right] d z^{\prime}\right)}-\beta_{\lambda_{0}}^{\text {mol }}(z)
\end{aligned}
$$

\subsection{Aerosol microphysical parameters retrieval}

The aerosol optical properties such as backscattering and extinction coefficient are closely related to their microphysics as shown in equation(5). $Q_{\text {ext }}$ and $Q_{\pi}$ stands for extinction and backscattering efficiency. $m$ stands for the complex refractive index. $n$ stands for the size distribution.

$$
\begin{aligned}
& \alpha(\lambda, z)=\int_{r_{\min }}^{r_{\max }} \pi r^{2} Q_{e x t}(r, \lambda ; m) n(r, z) d r \\
& \beta(\lambda, z)=\int_{r_{\min }}^{r_{\max }} \pi r^{2} Q_{\pi}(r, \lambda ; m) n(r, z) d r
\end{aligned}
$$

The Bayesian optimization method is applied to estimate the aerosol size distribution and refractive index as shown in equation (6).

$J=\sum_{i=1}^{n_{y}} \frac{\left[y_{i}-H(\mathbf{x})\right]^{2}}{\sigma_{y_{i}}^{2}}+\sum_{i=1}^{n_{x}} \frac{\left[x_{i}-b_{i}\right]^{2}}{\sigma_{b_{i}}^{2}}$

$J$ is the cost function. The forward model $H(\mathbf{x})$ predicts the observations from the state vector $\mathbf{X}$. Each observation $y_{i}$ is weighted by the inverse of its error variance $\sigma_{y_{i}}^{2}$. For the initial 
guess $b_{i}$, some elements of $\mathbf{X}$ are constrained by an a priori estimate. In the forward model, a three modes aerosol size distribution[4] is adopted. Each mode assumes a lognormal particle size distribution with two parameters.

\section{RESULTS}

\subsection{Aerosol optical properties}

Aerosol extinction coefficient profile retrieval is straightforward as described above. Fig. 3 shows an example for the retrieved extinction coefficient for $532 \mathrm{~nm}$ and $355 \mathrm{~nm}$, respectively and compared with two different retrieval methods.
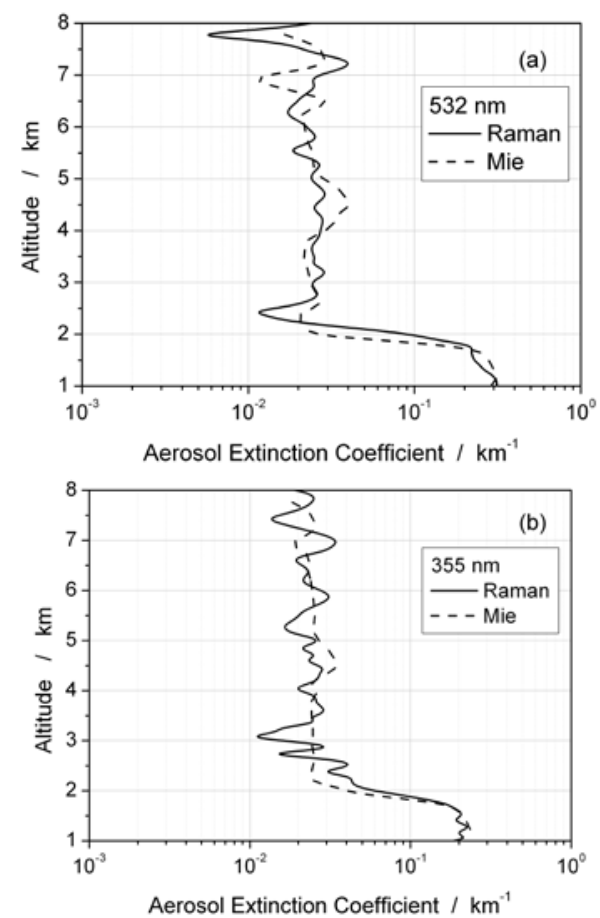

Fig 3 Aerosol extinction coefficient retrieved compared by Mie scattering and combined Raman technique (a) for $532 \mathrm{~nm}$ wavelength (b) for $355 \mathrm{~nm}$ wavelength

From the fig.3, one can see these two profiles coincided well, for each wavelength which indicated these two methods both worked correctly. Due to the differential process when applied the Raman technique the variation of the retrieved extinction coefficients is bigger than the Fernald algorithm.

\subsection{Cloud optical properties}

For the optical thin cirrus clouds which the lidar can penetrated through, applied the optical depth constrained method[5], the lidar ratio cloud be estimate precisely. The color ratio of different wavelength pairs could also been calculated. Based on two-year dataset obtained by TMPRL in Hefei, the backscattering color ratio was calculated and statistically analysed as shown in Fig.4. One can see the maximum occurrence is 0.9 , 0.7 and 0.6 for $1064 / 532,532 / 355$ and $1064 / 355$ wavelength pairs, respectively.
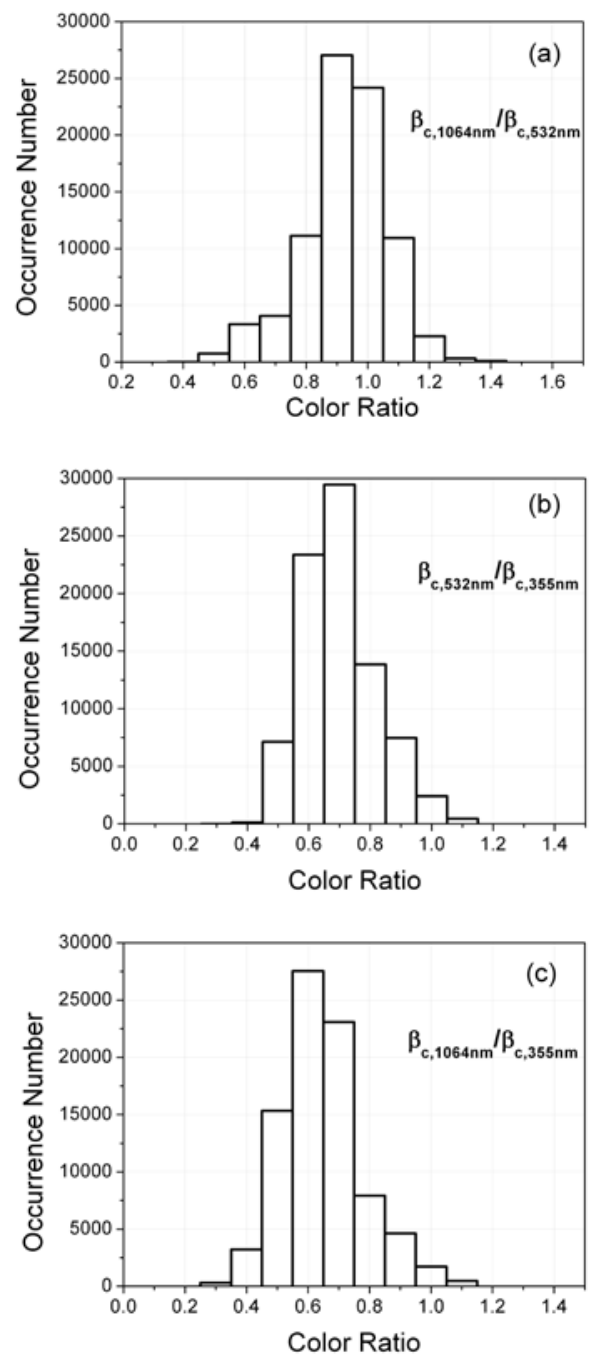

Fig.4 Statistics of the backscatter color ratio for three wavelength pairs (a) $1064 / 532 \mathrm{~nm}$ (b) $532 / 355 \mathrm{~nm}$ (c) $1064 / 355 \mathrm{~nm}$

For estimating the shape of the ice crystal of these cirrus clouds, the ray tracing method is applied to simulate the backscatter color ratio of six types[6], i.e., aggregate, hollow column, plate, bullet rosette, dendrite and solid column. Three of them is shown in the fig.5. 

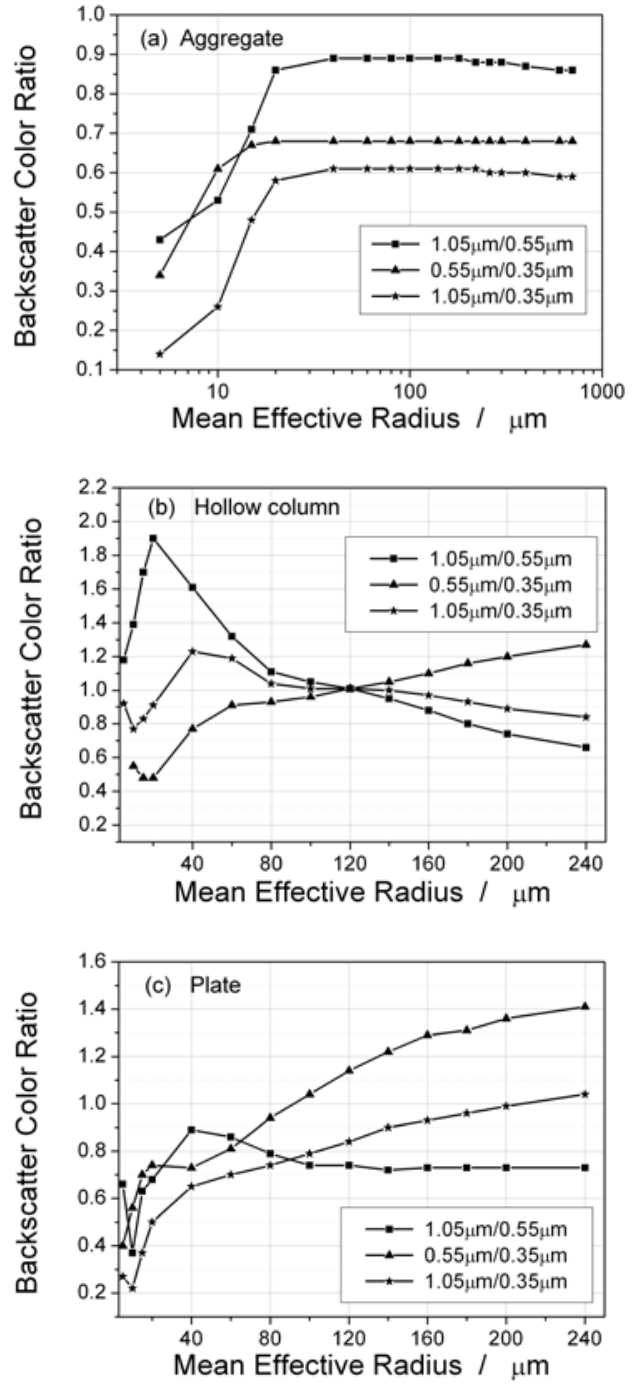

Fig.5 Simulated backscatter color ratio of three wavelength pairs for different shape of ice crystal (a) aggregate (b) hollow column (c) plate

From fig.5(a), one can see the backscatter color ratio kept stable on $0.6,0.7$ and 0.9 when the mean effective radius greater than $20 \mathrm{um}$ for $1.05 / 0.35,0.55 / 0.35$ and $1.05 / 0.55$ um wavelength pairs, respectively. It could be infer that most ice crystal observed in fig.4 seemed to be aggregate shape with mean effective radius greater than 20um.

\subsection{Aerosol microphysical properties and validation}

In August 2013, TMPRL was shipped to Wenshui city in Shanxi province which was about 1000km far from Hefei to join the field campaign to study the aerosol and cloud vertical properties and their interaction. A PCASP instrument was carried on an airplane to measure the vertical structure of the aerosol size distribution over the location of TMPRL. The retrieved results are shown in fig.6. The trend of these two profiles looks reasonable. The effective radiuses measured by PCASP were larger than the retrieval by TMPRL due to the PCASP measuring the aerosol radius greater than $0.2 \mathrm{um}$. The TMPRL algorithm needs be revised to match the size range of PCASP. More detail results will be done and shown in the conference.

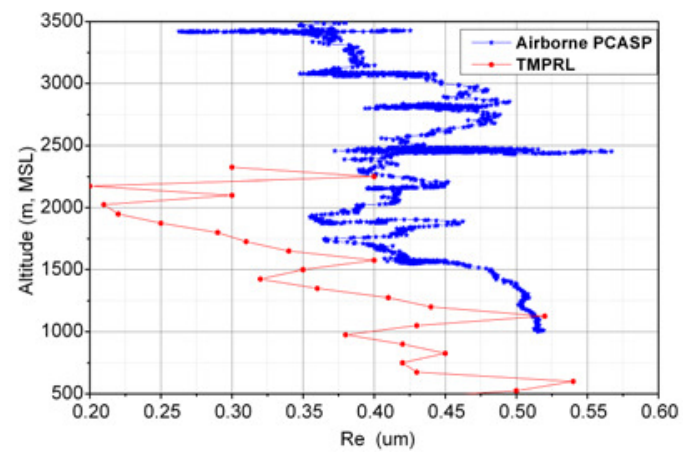

Fig.6 Aerosol effective radius retrieved by TMPRL compared with Airborne measurement

\section{CONCLUSIONS}

A movable multi-wavelength Raman lidar was built to study both optical and microphysical properties of aerosol and cloud. The first attempt to validate the aerosol vertical size distribution with airborne measurement was done and shown promising. More detail works need to be elaborated in the future, including the robust retrieval algorithms and more validation field campaign.

\section{ACKNOWLEDGEMENTS}

This work was supported by the National Natural Science Foundation of China under Grant No. 41075016 and National Basic Research Program of China under Grant No. 2013CB955802.

\section{REFERENCES}

[1] IPCC AR5, Summary for Policymakers. (2013)

[2] F. G. Fernald, Appl. Opt. 23, 652-653 (1984)

[3] A. Ansmann, et al. Applied Physics B 55(1): 18-28 (1992)

[4] G. Chen, et.al. Atmospheric Chemistry and Physics, Volume 10, Issue 5, pp.13445-13493 (2011)

[5] S. A. Young, Appl. Opt., 34, 7019-7031 (1995)

[6] Z. Tao, et al., Chinese Optics Letters, 10(5), p050101, (2012) 